\title{
La problemática del médico serumista en el Perú: conclusiones de la Segunda Convención Nacional de Médicos Serumistas, 2013
}

The problem of Peruvian doctors doing rural service: conclusions of the Segunda

Convención Nacional de Médicos Serumistas 2013

\author{
Fiorella Inga-Berrospi $i^{1,2, a, b}$, Álvaro Taype-Rondán ${ }^{2, a}$, Nelson Purizaca-Rosillo ${ }^{3, c}$ \\ 'Consejo Regional de Lima III - Colegio Médico del Perú, Lima, Perú. \\ ${ }^{2}$ Universidad de San Martín de Porres, Lima, Perú. \\ ${ }^{3}$ Universidad Nacional de Piura, Piura, Perú. \\ ${ }^{a}$ Médico Cirujano; ${ }^{b}$ Delegada Nacional de Médicos SERUMS 2013; ${ }^{\circ}$ Interno de Medicina Humana.
}

An Fac med. 2014;75(3):271-2 / doi: http://dx.doi.org/10.15381/anales.v75i3.9785

SEÑOR EDITOR:

En el año 1981 nace en Perú el Servicio Rural y Urbano Marginal de Salud (SERUMS), como un programa de servicio a la comunidad en el primer nivel de atención, en el que participan profesionales de la salud titulados, colegiados y hábiles para el ejercicio de la profesión. Actualmente, el SERUMS es el principal instrumento del sistema de salud peruano en la búsqueda de una adecuada dotación de profesionales de salud en las poblaciones vulnerables. Logrando, entre otras cosas, que la mortalidad materna disminuya significativamente en algunos lugares del Perú ${ }^{(1)}$, y que la cobertura de personal de salud en distritos de extrema pobreza aumente de $52 \%$ a $89 \%{ }^{(2)}$.

A pesar de los beneficios que brinda el SERUMS a la población, este programa podría estar atentando contra los derechos laborales de los profesionales que lo realizan (serumistas). En ese contexto se ha cuestionado la obligatoriedad del programa (pues según la Ley 23330 es un requisito para laborar en el sector Público en la condición de nombrados, contratados o por servicio no personales; así como para realizar programas de segunda especialización), el retraso o incumplimiento de los pa- gos reglamentados, las deficiencias en el equipamiento brindado, la deficiente cobertura de aseguramiento ${ }^{(3)}$ y la inseguridad de su ejercicio. Este último punto es uno de los más preocupantes, puesto que se han reportado incluso casos de incapacidad física y muerte entre los serumistas ${ }^{(4,5)}$.

Ante esta situación, el Comité del Médico Joven del Colegio Médico del Perú viene implementando, desde el año 2006, una serie de acciones que buscan velar por los médicos serumistas ante los peligros que afrontan en el ejercicio de su labor. Estas incluyen la organización de cursos de inducción al SERUMS por regiones, de convenciones nacionales y regionales de médicos serumistas y la implementación de un sistema de delegados regionales de SERUMS (quienes asumen el compromiso de velar por el bienestar de sus pares en cada región).

De esta manera, durante el 2013 se realizaron trece convenciones regionales de médicos serumistas en doce regiones del Perú, con el objetivo de generar espacios de debate acerca de la problemática del SERUMS. Asimismo, el 8 y 9 de noviembre de 2013 se llevó a cabo en Lima la Segunda Convención Nacional de Médicos Serumistas, con participación de médicos serumistas de diversos departamentos del Perú, así como 18 delegados regionales de SERUMS, representantes de la Oficina de Recursos Humanos del Ministerio de Salud (MINSA) y del Comité Nacional de Residentado Médico. En dicho evento se debatieron los problemas recurrentes en la práctica del SERUMS, y se realizó un consenso de las recomendaciones principales, que se exponen en la tabla 1.

La problemática del SERUMS en el Perú se encuentra lejos de encontrar solución. Sin embargo, las convenciones de médicos serumistas resultan ser una iniciativa importante para obtener recomendaciones realistas al respecto. Por lo tanto, es necesario que próximas convenciones tengan mayor participación de las instituciones prestadoras de servicios (como MINSA, EsSalud, Sanidades FFAA, PNP, Ejército del Perú y Sector Privado), así como de la comisión de Salud del Congreso de la República; y que sus conclusiones finales sean enviadas a las instituciones pertinentes mediante comunicados oficiales.

\section{REFERENCIAS BIBLIOGRÁFICAS:}

1. Dayrit MM, Dolea C, Dreesch N. Abordando la crisis de los Recursos Humanos para la Salud: ¿Hasta dónde hemos llegado? ¿Qué podemos esperar lograr al 2015? Rev peru med exp salud publica. 2011;28(2):327-36 
2. Arroyo J, Hartz J, Lau M. Recursos Humanos en Salud al 2011: evidencias para la toma de decisiones. [Internet]. Lima: Ministerio de Salud; 2011. Recuperado a partir de: http://www.bvsde.paho. org/texcom/cd046043/serieRHUS14.pdf

3. Mejia CR, Quiñones-Laveriano DM, Espinoza KG, Quezada-Osoria C. Deficiente cobertura de aseguramiento a médicos durante el servicio rural y urbano-marginal en Perú. Rev peru med exp salud publica. 2013;30(2):220-3.

4. Galán-Rodas E, Diaz-Vélez C, Villena J, Maguiña C. Mortalidad de médicos que realizan el servicio rural (SERUMS) en Perú, 2006-2009. Rev peru med exp salud publica. 2010;27(3):483-4.

5. Wong P. Los nuevos mártires de la medicina en el Perú. An Fac med. 2009;70(2):151-2.
Carta recibida el 25 de abril de 2014

Correspondencia:

Dr. Álvaro Taype-Rondán

C.S. Cajacay, Ancash, Perú

Universidad de San Martín de Porres, Lima, Perú

Teléfonos: 970001517 / RPM: \#970 001517

Correo electrónico: alvaro_athos@hotmail.com

Tabla 1. Propuestas priorizadas en la Il Convención Nacional de Médicos Serumistas, 2013.

1. Evaluar y reforzar adecuadamente a los coordinadores regionales y los comités regionales/subregionales SERUMS del MINSA, en cumplimiento del capítulo VI - artículo 19 de la Ley del SERUMS N ${ }^{2} 2330$, haciendo énfasis en su función de mantener coordinación permanente con los serumistas para optimizar el programa SERUMS.

2. El MINSA debe regular y articular con los gobiernos regionales, a fin de velar por el bienestar de los médicos serumistas fomentando la capacitación continua, respetando la ley del trabajo médico, sus derechos y deberes.

3. Generar información sobre las plazas ofertadas en los procesos SERUMS y sus condiciones actuales a través de un instrumento de recolección de datos denominado Observatorio Nacional de SERUMS, impulsado por el Colegio Médico del Perú en el año 2012.

4. Mejorar la Comunicación del MINSA con los profesionales SERUMS a través de la página oficial "Comunícate SERUMS", la cual debe contener información actualizada y real de cómo se lleva a cabo el programa SERUMS en cada región del país así como publicar los informes del SERUMS del proceso anterior.

5. Concientizar a la Coordinación SERUMS del MINSA sobre la importancia de las convenciones de médicos serumistas, de manera que se garantice la participación de autoridades, responsables y coordinadores SERUMS en dichos eventos. Asimismo, las conclusiones deben ser consideradas en la Reforma del SERUMS.

6. Valorar la labor y aporte del Médico SERUMS como profesional clave para el desarrollo del sistema de salud significando la representación y la primera respuesta del gobierno para afrontar los problemas de salud de las poblaciones más vulnerables y empobrecidas de nuestro país.

7. Exigir la revisión del listado de los establecimientos de salud ubicados en zonas Alejadas y de Frontera aprobado por Resolución Ministerial No 190-2013/ MINSA que cuentan con bonificación mensual a favor del personal de salud que labora en dichos establecimientos, debido al reporte de establecimientos que debieran estar incluidos en dicho listado.

8. Solicitar el cumplimiento del Artículo 17 de la Ley SERUMS №2330 la cual estipula como deber del Comité SERUMS del MINSA proponer políticas de capacitación e información dirigidas a los profesionales, enfatizando en la continuidad de las mismas.

9. Supervisar los casos especiales en el proceso de adjudicación de las plazas del SERUMS a nivel nacional.

10. Establecer lineamientos con respecto a una normativa que regule los días libres, a favor del bienestar del médico SERUMS.

11. Solicitar a los Consejos Regionales del Colegio Médico del Perú la participación activa y el compromiso permanente con los representantes de los médicos SERUMS en las regiones a fin de velar y supervisar el cumplimiento del programa SERUMS en condiciones favorables para los miembros de la orden.

12. Fortalecer los cursos de Inducción al SERUMS organizados por los Consejos Regionales del Colegio Médico del Perú dando principal énfasis en áreas como atención primaria de la salud, legislación médica y gestión sanitaria.

13. Solicitar al Colegio Médico del Perú capacitaciones virtuales periódicas que orienten a la solución de problemas Médicos Legales en el SERUMS.

14. Exigir a las Instituciones involucradas en la formación médica en pre grado que se implementen cursos de Idiomas como el quechua y aymara como parte de la currícula universitaria.

15. Considerar la importancia del Internado Rural, de los cursos de gestión en salud y salud pública para el adecuado desarrollo del programa SERUMS y el trabajo en el primer nivel de atención en salud.

16. Generar evidencia científica para describir la problemática actual del SERUMS y evaluar alternativas de solución.

17. Exigir al MINSA la evaluación de las plazas de los procesos SERUMS I - II, a fin de ofertar solo aquellas que cuenten con condiciones favorables y de seguridad para el ejercicio de la profesión médica. 